\title{
Preparation, characterization and dielectric behaviour of some yttrium doped strontium stannates
}

\author{
P K BAJPAI*, KULDEEP RATRE, MUKUL PASTOR and T P SINHA** \\ School of Pure and Applied Physics, Guru Ghasidas University, Bilaspur, Chattisgarh 495 009, India \\ **Bose Institute, Kolkata 700 009, India
}

MS received 22 July 2002

\begin{abstract}
Samples of $\mathrm{Sr}_{(1-3 x / 2)} \mathrm{Y}_{x} \mathrm{SnO}_{3}$ are prepared by usual solid state reaction route. X-ray diffraction studies confirm the formation of single cubic perovskite single phase. The dielectric constant and dielectric loss at $1 \mathrm{kHz}$ were measured in the temperature range from room temperature up to $\cong 150^{\circ} \mathrm{C}$. The dielectric constant decreases and losses increase with increased yttrium content in the samples. The percentage porosity and unit cell parameters are also calculated for the samples.
\end{abstract}

Keywords. Electronic ceramics; sensors; dielectric constant; strontium stannate.

\section{Introduction}

Sensors have become important devices because they provide information about physical and chemical signals, which could otherwise be difficult to perceive directly. $\mathrm{BaSnO}_{3}$ and $\mathrm{SrSnO}_{3}$ have been widely investigated as sensor materials along with their solid solutions with $\mathrm{BaTiO}_{3}$ (Sze et al 1998). In semiconductor sensors, the adsorption of gases on the surface of the material can bring significant change in the electrical resistance of the material. The mechanism of semiconductor gas sensors can be distinguished in two different classes. The first involves the change in the surface conductance while the second involves change in bulk conductance. The materials, which sense small concentration of reactive gases in air, rely by the change in the surface conductance and detect the displacement from equilibrium condition.

This class of compounds having cubic perovskite structure $\left(\mathrm{CaTiO}_{3}, \mathrm{SrSnO}_{3}, \mathrm{BaSnO}_{3}\right.$, etc) show paraelectric behaviour at room temperature and are found to be semiconductors exhibiting relaxor behaviour when doped with more than $1 \% \mathrm{Y}_{2} \mathrm{O}_{3}$ and sintered in an inert atmosphere at very low oxygen partial pressure (Neirman and Burn 1984). The same compound, when sintered in an oxygen rich atmosphere, exhibits dielectric behaviour similar to pure compound (Buchanan 1986). When $\mathrm{Y}^{3+}$ ions are substituted at $\mathrm{A}$ site of $\mathrm{SrSnO}_{3}$, two types of changes are expected. The ionic size will be affected resulting into change in the unit cell dimension. Secondly, substitution of $\mathrm{Y}^{3+}$ ions at $\mathrm{A}$ site (divalent site) will not bring the charge neutrality, and electrical conductivity is expected to increase.

\footnotetext{
*Author for correspondence
}

The ceramic compounds are sintered to approach the equivalent single crystal behaviour as far as possible. Sintering increases density and in some cases it may reach up to $99.9 \%$. However, for sensor materials, loosely sintered particles or thin films give better response as it increases the effective surface area and/or makes gas particle percolation into bulk easy. Therefore, one of the objectives of material modification for sensors is to increase porosity of the material. Further, earlier reports on charge compensated perovskites show sintering in a nitrogen atmosphere, oxygen lost from these ceramics according to relation, $\mathrm{O}_{0}=1 / 2 \mathrm{O}_{2}+V_{0} \pm 2 e^{\prime}$ (Prakash et al 1987). On cooling from the firing temperature, reoxidation of the product takes place. However, sufficient time is not available for the reoxidation of the grains. Only grain boundaries are reoxidized and become insulating, thus enveloping the semiconductor grains. This gives rise to barrier layer at grain/grain boundary interfaces imparting very high dielectric constant to the resulting material. One therefore expects high dielectric constant and porosity in doped stannates.

In this paper, we report the preparation, structural characterization and dielectric behaviour of $\mathrm{Sr}_{(1-3 x / 2)} \mathrm{Y}_{x} \mathrm{SnO}_{3}$ ceramics for $x=0.0,0.01,0.03$ and 0.05 with a view to develop newer materials for semiconductor sensors.

\section{Experimental}

The starting materials, $\mathrm{SrCO}_{3}$ (SD fine, 99\%), $\mathrm{SnO}_{2}$ (BDH, 99.5\%) and $\mathrm{Y}_{2} \mathrm{O}_{3}$ (Indian Rare Earth, 99.9\%) were mixed together in appropriate molar ratio as per the equation

$$
(1-3 x / 2) \mathrm{SrCO}_{3}+\mathrm{SnO}_{2}+x \mathrm{Y}_{2} \mathrm{O}_{3} \rightarrow \mathrm{Sr}_{1-3 x / 2} \mathrm{Y}_{x} \mathrm{SnO}_{3}+\mathrm{CO}_{2}
$$


with $x$ being $0,0.01,0.03$ and 0.05 , respectively. The finely grinded powders were kept for calcination in the silicon carbide furnace at $1200^{\circ} \mathrm{C}$ for $24 \mathrm{~h}$. The calcined powders were homogenized and again calcinated at $1225^{\circ} \mathrm{C}$ for $16 \mathrm{~h}$. The finally calcinated powders were finely grinded and used to make pellets of $10 \mathrm{~mm}$ diameter and 1-2 mm thickness and were sintered at $1275^{\circ} \mathrm{C}$ for $24 \mathrm{~h}$. Sintered pellets were polished and electroded with silver conducting paint. These pellets were used for dielectric measurement. The calcined powder was characterized by $\mathrm{XRD}$ using $\mathrm{Cu}-\mathrm{K} \alpha \mathrm{X}$-ray radiation. The XRD pattern is shown in figure 1 , which was indexed on the basis of cubic perovskite structure (table 1). The indexed peaks clearly show single-phase formation. The lattice parameter deduced from XRD data are given in table 2 . The data show that $\mathrm{Y}^{3+}$ doping decreases the lattice constant. The dielectric measurements were performed at $1 \mathrm{kHz}$ using HP-4263B LCR meter and a home made temperature dependent measurement setup from room temperature up to $150^{\circ} \mathrm{C}$.

\section{Results and discussion}

From the capacitance data, the dielectric constant at each temperature is calculated using

$$
\varepsilon=C \cdot d / \varepsilon_{0} A,
$$

where $C$ is capacitance, $D$ the thickness and $\varepsilon_{0}$ the free space permittivity and $A$ the area of the pellet. The temperature dependence of dielectric constant and dielectric loss for various $\mathrm{Y}^{3+}$ doped $\mathrm{Sr}_{1-3 x / 2} \mathrm{Y}_{x} \mathrm{SnO}_{3}$ samples are shown in figures 2 and 3 , respectively. The frequency dependent dielectric behaviour is shown in figures 4 and 5. From figure 2, it is clear that dielectric constant increases with yttrium substitution and remains almost constant in the entire temperature range studied. This indicates that the vacancies created due to inherent charge compensation are not fully reoxidized and grain/ grain boundary interface is giving boundary layer capacitance, thus making them suitable materials for barrier layer capacitor application. The dielectric loss increases with temperature and the increase is almost exponential. This shows that the conductivity contribution is mainly due to hopping process. The frequency dependence of dielectric behaviour (figures 4-5) shows no dielectric dispersion. An important effect of $\mathrm{Y}^{3+}$ doping in these

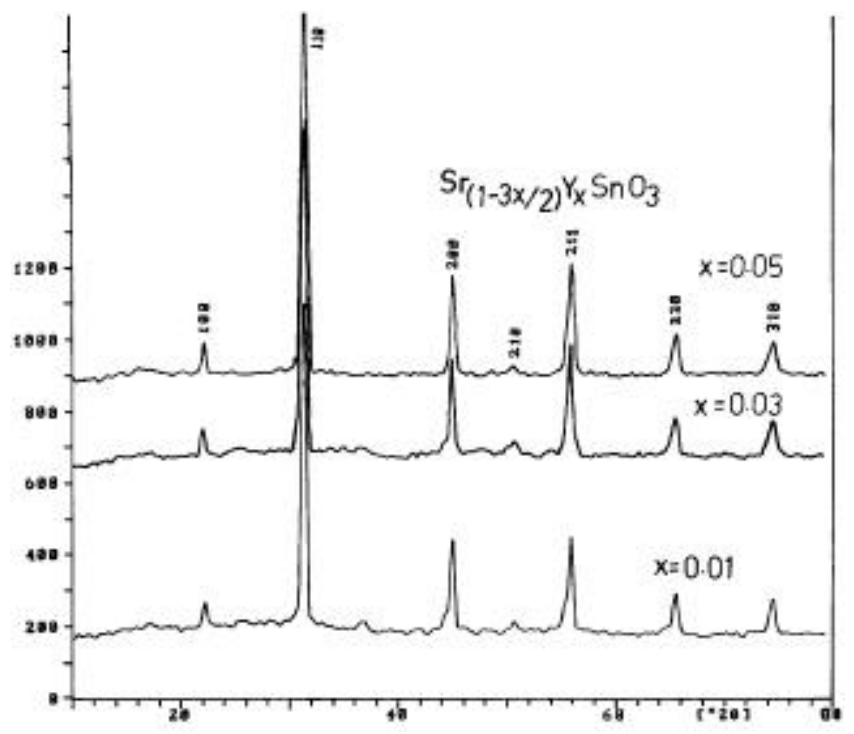

Figure 1. XRD peaks observed in doped systems.

Table 1. X-ray diffraction data for yttrium doped strontium stannates.

\begin{tabular}{|c|c|c|c|c|c|c|c|c|}
\hline \multicolumn{3}{|c|}{$x=0.01$} & \multicolumn{3}{|c|}{$x=0.03$} & \multicolumn{3}{|c|}{$x=0.05$} \\
\hline$d$-values & $I / O$ & $h k l$ & $d$-values & $I / O$ & $h k l$ & $d$-values & $I / O$ & $h k l$ \\
\hline $4 \cdot 0126$ & $8 \cdot 7$ & 100 & 4.0081 & $8 \cdot 7$ & 100 & 4.0028 & $9 \cdot 3$ & 100 \\
\hline $2 \cdot 8422$ & $100 \cdot 0$ & 110 & $2 \cdot 8342$ & $100 \cdot 0$ & 110 & $2 \cdot 8373$ & $100 \cdot 0$ & 110 \\
\hline $2 \cdot 0120$ & 27.7 & 200 & $2 \cdot 0086$ & $25 \cdot 1$ & 200 & 2.0063 & $22 \cdot 5$ & 200 \\
\hline 1.8044 & $2 \cdot 0$ & 210 & 1.7935 & $3 \cdot 3$ & 210 & 1.7968 & 2.5 & 210 \\
\hline 1.6434 & $30 \cdot 3$ & 211 & 1.6394 & $26 \cdot 6$ & 211 & 1.6390 & $23 \cdot 2$ & 211 \\
\hline 1.4240 & $9 \cdot 3$ & 220 & 1.4218 & 9.4 & 220 & 1.4219 & $8 \cdot 5$ & 220 \\
\hline 1.2729 & $7 \cdot 1$ & 310 & 1.2692 & $8 \cdot 2$ & 310 & 1.2710 & $6 \cdot 7$ & 310 \\
\hline
\end{tabular}

Table 2. Lattice constant, density data and percentage porosity for doped systems.

\begin{tabular}{lcccc}
\hline $\begin{array}{l}\text { Sample doping } \\
\text { percentage }(x)\end{array}$ & $\begin{array}{c}\text { Experimental } \\
\text { density }\left(\mathrm{g} / \mathrm{cm}^{3}\right)\end{array}$ & $\begin{array}{c}\text { Theoretical } \\
\text { density }\left(\mathrm{g} / \mathrm{cm}^{3}\right)\end{array}$ & $\begin{array}{c}\% \\
\text { Porosity }\end{array}$ & Lattice constant \\
\hline $0 \cdot 0$ & 4.98 & 6.4868 & 86 & 4.042 \\
$0 \cdot 01$ & 3.285 & 6.5330 & 79 & 4.040 \\
0.03 & 3.448 & 6.5340 & 68 & 4.023 \\
$0 \cdot 05$ & $2 \cdot 863$ & 6.5299 & 54 & 4.014 \\
\hline
\end{tabular}




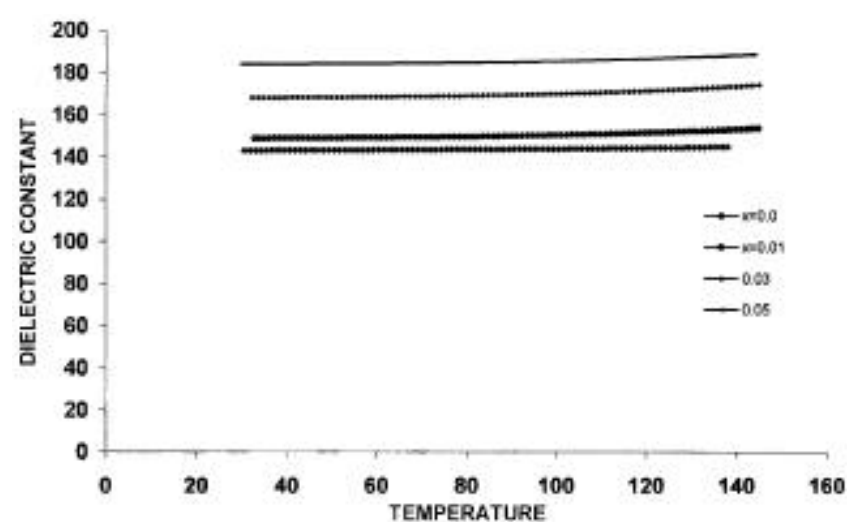

Figure 2. Dielectric constant as a function of temperature in $\mathrm{Sr}_{(1-3 x / 2)} \mathrm{Y}_{x} \mathrm{SnO}_{3}$.

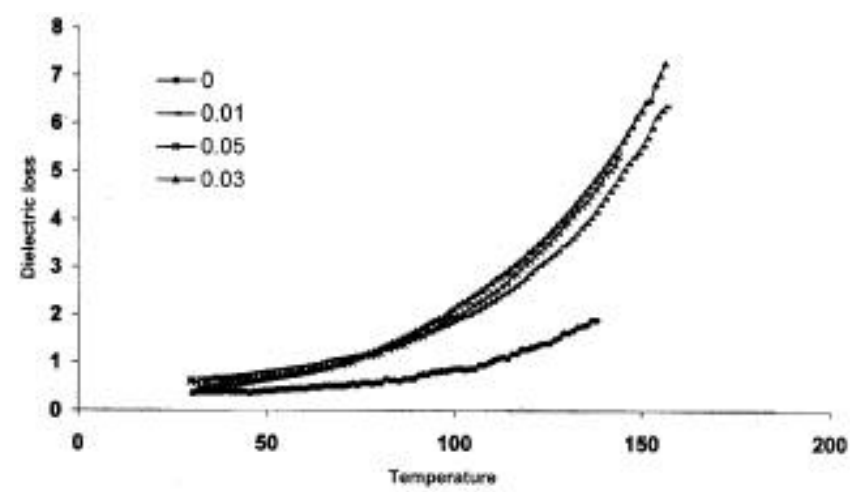

Figure 3. Dielectric loss as a function of temperature in $\mathrm{Sr}_{(1-3 x / 2)} \mathrm{Y}_{x} \mathrm{SnO}_{3}$.

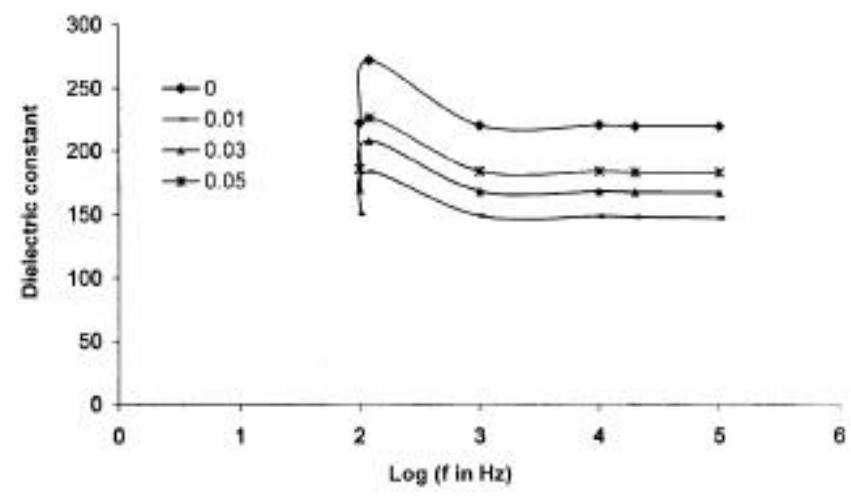

Figure 4. Effect of frequency on dielectric constant at room temperature in $\mathrm{Sr}_{(1-3 x / 2)} \mathrm{Y}_{x} \mathrm{SnO}_{3}$.

compounds is the change in their porosity. We have calculated the densities of different samples theoretically and compared them with measured densities as shown in table 2. Whereas, in the pure $\mathrm{SrSnO}_{3}$ pellets, the measured density being $\simeq 86 \%$ to that of theoretical density, it decreases with $\mathrm{Y}^{3+}$ substitution and becomes almost $50 \%$ in $5 \% \mathrm{Y}^{3+}$ substituted samples. The preparation conditions

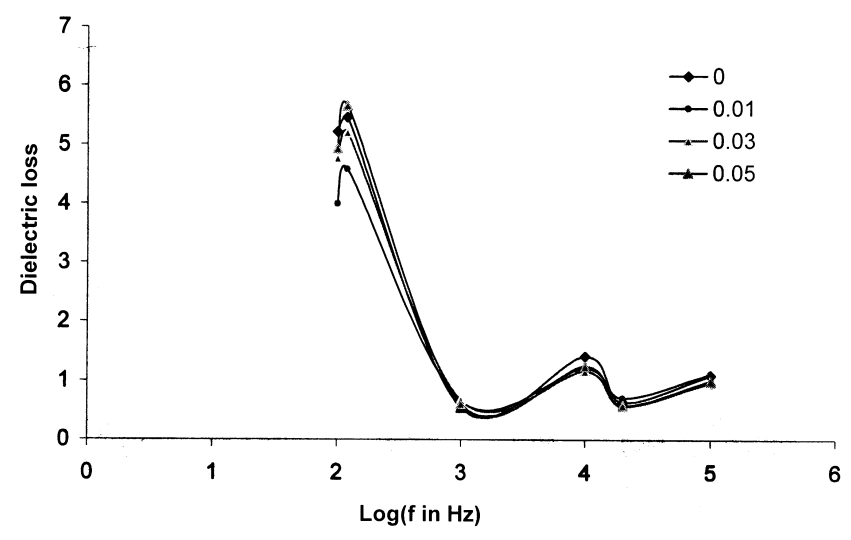

Figure 5. Effect of frequency on dielectric loss at room temperature in $\mathrm{Sr}_{(1-3 x / 2)} \mathrm{Y}_{x} \mathrm{SnO}_{3}$.

being similar for all samples indicate that the different ion size of $\mathrm{Y}^{3+}$ decreases packing density, therefore, increasing the porosity. This means that the doped material will have more absorption, giving better response when used as sensor materials. Measuring their water retention capacity further checks the increase in porosity. Pellets were socked in distilled water for $24 \mathrm{~h}$ and their weights were taken before and after water absorption. The results indicate increase in porosity with increasing $\mathrm{Y}^{3+}$ percentage in samples.

\section{Conclusions}

The present study shows that the inherent charge neutrality created by vacancies in the yttrium doped stannates makes them important materials from sensor application. These can be used as barrier layer capacitor. Also, the dielectric constant increases with A-site yttrium substitution and could be useful gas sensing material. In fact a number of systems with substitution of trivalent cation at $\mathrm{A}$ and B-sites simultaneously were studied earlier (Neirman and Burn 1984). However, the inherent charge compensated systems are very scant (Kulwfelti et al 1991).

The XRD results show that the prepared compounds have single phase. The lattice parameters calculated, show decrease in lattice constant with substitution. It is shown that dielectric constant and loss increases with $\mathrm{Y}^{3+}$ doping. The porosity factor also increases indicating that the yttrium-doped compound should be more sensitive for gas sensing in comparison to pure stannates. The frequency dependence of dielectric behaviour indicates dispersion free dielectric behaviour at audio frequencies.

\section{References}

Anderson J E and Graves Y B 1981 Semiconductor sensors (San Diego, CA: Academic Press) 
Buchanan R 1986 in Ceramic materials for electronic applications (New York: Dekker)

Kulwfelti B 1991 J. Am. Ceram. Soc. 74697

Neirman S and Burn J 1984 J. Mater. Sci. 19737
Prakash O, Prasad Ch D and Kumar D 1987 J. Solid State Chem. 69385

Sze S M 1988 VLSI technology (New York: McGrawHill) 